\title{
Frontières
}

\section{Résilience, spiritualité et réadaptation}

\section{Jocelyn Chouinard}

Volume 22, numéro 1-2, automne-printemps 2009-2010

Résilience et deuil

URI : https://id.erudit.org/iderudit/045032ar

DOI : https://doi.org/10.7202/045032ar

Aller au sommaire du numéro

Éditeur(s)

Université du Québec à Montréal

ISSN

1180-3479 (imprimé)

1916-0976 (numérique)

Découvrir la revue

\section{Citer cet article}

Chouinard, J. (2009). Résilience, spiritualité et réadaptation. Frontières, 22(1-2), 89-92. https://doi.org/10.7202/045032ar
Résumé de l'article

La spiritualité est l'un des facteurs qui permettent à l'être humain de donner du sens à sa vie. L'expérience de réadaptation à la suite d'un traumatisme ou d'une maladie peut devenir une occasion de transformation et de croissance spirituelle si elle s'inscrit dans le projet de vie de la personne. Le développement de l'outil Fil d'Ariane par le Centre de réadaptation Estrie a éte réalisé dans cette perspective. Le regard positif posé par l'intervenant peut permettre à la personne en réadaptation d'exprimer sa résilience, et par-delà, de s'adapter et de rebondir en dépit de l’adversité. d'utilisation que vous pouvez consulter en ligne.

https://apropos.erudit.org/fr/usagers/politique-dutilisation/ 


\section{Résumé}

La spiritualité est l'un des facteurs qui permettent à l'être humain de donner du sens à sa vie. L'expérience de réadaptation à la suite d'un traumatisme ou d'une maladie peut devenir une occasion de transformation et de croissance spirituelle si elle s'inscrit dans le projet de vie de la personne. Le développement de l'outil Fil d'Ariane par le Centre de réadaptation Estrie a été réalisé dans cette perspective. Le regard positif posé par l'intervenant peut permettre à la personne en réadaptation d'exprimer sa résilience, et par-delà, de s'adapter et de rebondir en dépit de l'adversité.

Mots clés: Résilience - spiritualité facteurs de protection - incapacités réadaptation - psychologie positive - mécanismes de coping.

\section{Abstract}

Spirituality is one of the factors that provide human beings with the resources to give meaning to their life. When integrated into an overall life project, the rehabilitation process undertaken after a trauma or an illness can become an opportunity for transformation and spiritual growth. With this in mind, the CRE developed a rehabilitation tool called the Fil d'Ariane (Ariadne's Clew). The caseworker's positive outlook can help a person who is undergoing a rehabilitation process demonstrate resilience and, eventually, adapt and pull through in the face of adversity.

Keywords: Resilience - spirituality protection factors - disabilities rehabilitation - positive

psychology - coping mechanisms.

\section{$\begin{array}{llllllllllll}\text { I } & N & T & E & R & V & E & N & T & \text { I } & O & N\end{array}$}

\section{RÉSILIENCE, SPIRITUALITÉ ET RÉADAPTATION}

Jocelyn Chouinard, M.A., psychologue, Centre de réadaptation Estrie.

Malgré son rôle pressenti dans les mécanismes d'adaptation, la résilience a été peu étudiée en réadaptation, comparativement au modèle déficience-incapacité-handicap (Bonanno, communication personnelle, janvier 2004). Emprunté au domaine de la physique des métaux, le concept a d'abord été proposé pour illustrer la capacité qu'ont certains enfants de rebondir et de s'adapter positivement dans des circonstances difficiles (Black et Lobo, 2008; Cyrulnik, 1999). En l'étendant au domaine de la psychologie adulte, on a suggéré qu'il jouait un rôle dans la récupération des personnes atteintes de cancer et du sida (Jacelon, 1997). D'autres l'ont proposé comme facteur pouvant expliquer comment des personnes qui vivent avec des incapacités depuis la naissance peuvent se développer malgré leur condition et trou- ver un sens à leur vie (King et al., 2003). Enfin, White et al. (2008) ont appliqué le concept à la réadaptation des traumatisés craniocérébraux.

Précurseur de l'approche cognitive en thérapie, le philosophe grec Épictète (cité par Seligman et Csikszentmihalyi, 2000) affirmait dans l'Antiquité que ce n'est pas l'événement en soi qui détermine le niveau de bonheur (ou de malheur) des gens, mais plutôt la façon dont ils interprètent ce qui leur arrive. Pourquoi certains individus réussissent-ils à s'adapter à leurs incapacités et à vivre heureux alors que d'autres en seront incapables? Comment interprètent-ils ce qui leur arrive? Comment réussissent-ils à maintenir un sens à la vie après un grave traumatisme ou l'annonce d'une maladie incurable? Est-ce parce qu'ils sont plus forts physiquement ou moralement? Est-ce que l'environnement joue aussi un rôle? Comment les 
intervenants de réadaptation peuvent-ils faciliter l'adaptation, voire la croissance personnelle de leurs usagers?

Cet article porte sur une dimension du concept de résilience, soit la spiritualité, appliquée au domaine de la réadaptation physique. Nous ferons d'abord état des définitions les plus courantes de la résilience et de celle que nous retenons concernant la spiritualité. Nous verrons ensuite comment cette dernière peut être considérée comme un facteur de résilience. Nous aborderons le cadre de référence auquel se rattachent les travaux actuellement en cours au Centre de réadaptation Estrie, puis nous terminerons par une illustration clinique.

\section{DÉFINITIONS DE RÉSILIENCE ET SPIRITUALITÉ}

La résilience est généralement définie comme étant la capacité de rebondir à la suite d'un événement malheureux (APA, 2010). Pour certains, elle dépend d'un ensemble de caractéristiques de la personnalité qui atténuent les effets du stress (Wagnild et Young, 1993), tandis que pour d'autres, elle est plutôt l'indice d'un processus dynamique où entrent en jeu des facteurs personnels et environnementaux (Dyer et McGuinness, 1996). Rutter (1985) parle même d'un continuum vulnérabilitérésilience opposant facteurs de risque à facteurs de protection.

Certains affirment qu'on peut la mesurer comme n'importe quel trait de personnalité, d'où la construction d'échelles comme le Resiliency Scale (Wagnild et Young, 1993) et le Connor-Davidson Resilience Scale (Connor et Davidson, 2003). À l'inverse, d'autres prétendent qu'il s'agit plutôt de comportements pouvant être appris et développés (APA, 2010).

Richardson (2002) considère qu'il s'agit d'une dimension beaucoup plus profonde qu'un trait. Il parle d'une énergie ou d'une force motivationnelle qui existe en chaque individu, et qui le pousse à s'actualiser dans les moments difficiles. L'adversité se pose alors comme une opportunité de déséquilibre activant les facteurs de protection et donnant à la personne une occasion de croissance. Selon Richardson, les professionnels de la santé peuvent stimuler cette croissance en aidant les usagers à découvrir ces «sous-produits positifs» d'un événement comme un traumatisme ou une maladie (McMillen et LovelandCook, 2003). Il deviennent alors ce que Boris Cyrulnik appelle des «tuteurs de résilience» (Cyrulnik, 2008).

Certains théoriciens du deuil (cités par Bonanno, 2004) croient que l'absence de détresse émotive à la suite d'une perte se produit chez l'individu froid et insensible qui se protège contre une trop grande angoisse (déni). Bonanno (2004) affirme au contraire que le résilient souffre émotionnellement et ressent de la tristesse comme n'importe quel individu à la suite d'un événement difficile. Ce qui le distingue, c'est le caractère occasionnel et transitoire de ces perturbations émotives, et le maintien d'un équilibre physique et psychologique relativement stable, qui lui permet de retrouver graduellement un état normal.

Comme le mentionne Bonanno, la résilience n'est pas l'apanage d'individus exceptionnellement forts physiquement et mentalement. La très grande majorité des gens qui ont été exposés à des traumatismes ou à des événements menaçant

«CELUI QUI A UN POURQUOI

QUI LUI TIENT LIEU DE BUT

PEUT AFFRONTER

\section{N'IMPORTE QUEL COMMENT »}

(Nietzche, cité par Frankl, 2006).

leur intégrité reprennent une vie normale sans présenter de symptômes chroniques. Citant les travaux de Galea et ses collaborateurs, il donne l'exemple récent des résidents de Manhattan dont 7,5\% répondaient aux critères du syndrome de stress post-traumatique du DSM-IV un mois après l'attaque terroriste du 11 septembre, contre $0,6 \%$ six mois plus tard.

La spiritualité est au cœur du cadre de référence de la pratique des ergothérapeutes au Canada (Law, 2002). Elle est définie simplement comme étant ce qui donne du sens à l'occupation humaine (travail, loisirs, relations interpersonnelles) et sous-tend une volonté ou une motivation intrinsèque à faire des choix et agir. Cette définition séculière est aussi partagée par les tenants de l'approche humaniste Planetree pour qui la spiritualité est ce qui donne à la personne la capacité de trouver du sens à ce qui lui arrive, notamment lorsqu'elle est confrontée à des problèmes de santé (Frampton et Charmel, 2009).

\section{RÉSILIENCE ET SPIRITUALITÉ}

La résilience est facilitée par la présence de certaines caractéristiques personnelles. L'individu qui a un fort sentiment de compétence personnelle et sociale ${ }^{1}$ est généralement capable de mieux s'adapter aux difficultés de sa vie. Le soutien de la famille ou de la communauté sont aussi reconnus comme facteurs de protection externes très efficaces contre les situations de vie difficiles (Black et Lobo, 2008; White et al., 2008).

La spiritualité, définie autour de la notion de sens à l'activité humaine, est considérée comme un important facteur de résilience. Celui qui cherche à trouver un sens à ce qui lui arrive a généralement plus de facilité à surmonter les écueils de la vie (Bonanno, 2004). À l'inverse, l'absence de sens mène à la détresse, voire au suicide (Yalom, 2008). S'inspirant des travaux du psychiatre autrichien Viktor Frankl, Thompson et ses collègues (2003) affirment que le fait de pouvoir donner un sens à sa vie représente la force la plus fondamentale qui dynamise chaque être humain et le rend capable de dépasser les contraintes et d'atteindre un niveau d'ajustement et d'accomplissement satisfaisant.

\section{UN CADRE DE RÉFÉRENCE POSITIF}

Le Processus de production du handicap (PPH) (Fougeyrollas, 1998) est le cadre de référence le plus largement utilisé dans les milieux de réadaptation au Québec. En évitant de mettre l'accent sur la maladie et l'incapacité, ce modèle suggère que les situations de handicap se produisent à la suite de l'interaction dynamique de facteurs appartenant à l'individu et à l'environnement. Ce nouveau paradigme a permis de mieux comprendre la genèse des situations de handicap et d'adopter des pratiques qui vont faciliter la reprise des habitudes de vie et la participation sociale.

Malgré son éclairage sur la contribution de l'environnement, le modèle du PPH n'explique pas comment l'individu s'adapte et se réadapte, allant même pour certains jusqu'à atteindre des niveaux d'actualisation de soi plus élevés qu'avant l'épisode d'incapacité. Dans un document inédit, Lachapelle (2009) souligne la nécessité de revenir à des notions de croissance personnelle ou d'actualisation de soi pour comprendre comment la personne s'adapte, et suggère de reconsidérer "nos façons d'intervenir auprès de la personne handicapée vivant avec une déficience (p. 5)».

En cherchant à comprendre les mécanismes de résilience, la psychologie positive nous offre un point de vue intéressant à cet égard (Dunn et Dougherty, 2005). À la base, elle postule que toute personne est capable d'agir sur son bien-être et sur sa santé. Plutôt que de considérer uniquement les facteurs dits défavorables ${ }^{2}$, on propose de rechercher ceux qui vont permettre à la personne d'affronter avec succès ses défis, et qui font que sa vie a du sens et vaut d'être vécue (Pledger, 2003 ; Seligman et Csikszentmihalyi, 2000). Tout 
en tenant compte de «l'interaction dynamique de facteurs appartenant à l'individu et à l'environnement ", cette école de pensée invite à se demander comment une situation de handicap peut se transformer en une situation de développement personnel. L'épisode de maladie ou d'incapacité cesse alors d'être vu comme un échec et devient plutôt une opportunité de croissance (Black et Lobo, 2008; White et al., 2008). La découverte de sens peut ainsi éviter l'apparition de symptômes de détresse psychologique (Frankl, 2006).

\section{UNE EXPÉRIENCE \\ DE TRANSFORMATION}

La réussite de la réadaptation sera intimement liée au sens que la personne pourra donner à cet épisode de vie dans son cheminement personnel. Pour paraphraser Frankl, il s'agit moins de trouver un sens à la maladie ou au traumatisme, que de chercher à donner un sens à la vie «à chaque jour et à chaque heure» (Frankl, 2006). Comment transformer les défis quotidiens que j'affronte dans la réalisation de mes habitudes de vie, en des occasions de croissance et de développement? Comment trouver du sens aux rôles et aux occupations qui seront désormais les miens? Comment comprendre que j'ai toujours ma place dans la société et que ma vie vaut la peine d'être vécue?

Le défi de l'intervenant en réadaptation sera d'aider l'usager à découvrir les «sousproduits positifs » de sa condition à travers des nouvelles façons de réaliser ses habitudes de vie. En d'autres termes, devenir un tuteur de sa résilience et faire de sa réadaptation une expérience de transformation et d'actualisation, un point tournant dans son projet de vie (King et al., 2003).

Ces questions et ces défis nous ont amenés à introduire progressivement des interventions touchant la dimension spirituelle dans nos soins de réadaptation. Ainsi, nous avons développé un outil que nous avons appelé Le Fil d'Ariane (Chouinard et Tardif, 2005). Il s'agit d'un cadre d'entrevue semi-dirigée où nous invitons l'usager à se raconter à partir de différents thèmes : son enfance et son adolescence, ses motivations de fond, les défis qu'il a affrontés, ses expériences de transformation, ses aspirations, les connexions qui le relient à autrui, à la nature, à un être suprême. Cette narration le place au centre de sa propre vie et lui donne l'occasion de saisir les liens qui peuvent exister entre son passé, le présent et ce que pourrait être son avenir au sortir de la réadaptation (Mattingly, 2007). C'est aussi une occasion d'apprendre sur lui-même et d'iden- tifier les ressources internes et externes qui l'ont aidé à traverser d'autres épisodes difficiles de sa vie auparavant. L'entrevue est enregistrée (support visuel ou auditif) et l'enregistrement remis à l'usager s'il le désire.

Le contenu de l'entrevue est analysé par le professionnel de réadaptation qui tente de dégager les facteurs positifs de sa personnalité et de son histoire, mais surtout le fil conducteur qui a pu guider l'usager dans ses choix et expériences antérieurs. En adoptant un regard délibérément positif et porteur d'espoir, il rédige ensuite un récit mettant en lumière ce fil conducteur et tous les facteurs favorables à la réadaptation et à la croissance de l'usager. Ce récit est validé par l'usager et présenté à ses proches et aux professionnels de l'équipe de réadaptation. Ceux-ci vont tenter d'inscrire leurs interventions dans l'optique et la continuité du Fil d'Ariane, donnant ainsi plus de sens à leurs interventions thérapeutiques.

\section{HISTOIRE DE CAS}

\section{CONTEXTE CLINIQUE}

Mathilda est une jeune femme âgée de 35 ans $^{3}$ qui a été admise au Programme de traumatologie du Centre de réadaptation Estrie environ six mois après avoir été victime d'un accident de voiture. Depuis cet accident, elle se sent très différente: elle n'est pas déprimée, mais elle a l'impression de tourner en rond, se sent désorganisée, sans but ni attentes: elle est là parce qu'on lui a dit que ça lui ferait peut-être du bien d'aller en réadaptation.

\section{L'HISTOIRE DE MATHILDA}

Le contenu de l'entrevue Fil d'Ariane a permis de mettre en lumière, à travers une histoire d'abus, de négligence et de violences à répétition, des forces et une ligne directrice qui pourraient être mises à profit pour faciliter une adaptation positive.

D'abord, Mathilda est débrouillarde. Elle l'a prouvé pendant son enfance lorsqu'elle usait de divers stratagèmes pour se protéger et protéger ses sœurs de la violence de son père. De plus, l'école lui a appris qu'elle est intelligente et vive. Elle y a vécu ses premières réussites et réalisé que tous les adultes ne sont pas des abuseurs.

Mathilda a appris qu'elle peut faire confiance: quelque part pendant son secondaire, elle déballe un après-midi durant toute son histoire d'abus à une enseignante qui l'écoute sans la juger. Cette rencontre est un point tournant de sa vie: après avoir dérapé pendant ses premières années de secondaire, elle retrousse ses manches et affronte avec courage et détermination les années d'études qui vont lui ouvrir les portes de l'université.

C'est à ce moment qu'elle découvre sa vocation d'aidante: elle choisit d'étudier le phénomène de la violence faite aux enfants afin de faire en sorte que moins de petites filles aient à subir les comportements violents des adultes. Presque au fil d'arrivée, elle abandonne ses études en psychoéducation: une grossesse non désirée, un conjoint violent et plusieurs démêlés avec les policiers et la DPJ vont la précipiter dans un nouveau cauchemar qui la mènera cette fois jusqu'à la tentative de suicide.

C'est au cours d'une lente remontée qu'elle reprendra contact avec sa mère. Plusieurs mois à reconstruire ses forces, à refaire un tant soit peu confiance à la vie, à entreprendre un dialogue avec sa mère. Plusieurs mois à réapprendre à fonctionner, à se débrouiller avec les comptes, l'épicerie, le ménage, et, surtout, à se défaire de l'emprise de son ex-conjoint et à prouver à la DPJ qu'elle peut encore être une bonne mère malgré ses antécédents.

\section{LE FIL D'ARIANE DE MATHILDA}

Voici le récit que présente l'intervenante à Mathilda :

Ton histoire, Mathilda, c'est l'histoire du courage, de la ténacité et de la persévérance. C'est l'histoire d'une toute petite fleur qui s'accroche au rocher, malgré les vents et les fortes pluies. C'est l'histoire d'une petite fille qui a choisi de défier son père et sa destinée, pour s'accrocher à un rêve et devenir quelqu'un.

$\mathrm{Tu}$ as appris pendant ton enfance à devenir débrouillarde: lorsque tu t'enfuyais de la maison avec ton chien pour te réfugier dans la forêt, où tu savais que ton père ne pouvait te rejoindre. Ce chien qui est devenu ton confident, et avec qui tu as appris la parole.

L'école t'a révélé ta valeur et la force intérieure qui t'animait. La présence chaleureuse des enseignantes, la fierté que tu pouvais lire dans leurs yeux, leurs encouragements à poursuivre tes études, tout ça était tellement contraire à l'image que ton milieu familial te renvoyait, que tu avais même du mal à y croire. À un point tel que tu as décidé de faire passer un test de confiance à tous ces adultes : quelques années de décrochage au secondaire, jusqu'à ce qu'Hélène, une ancienne enseignante du primaire, reconnaisse en toi la petite fille à qui elle aimait tant enseigner. Cet 
après-midi-là, tu as repris contact avec la parole libératrice. C'est au cours de cette rencontre que naquit ton projet de vie: tu allais devenir celle qui empêcherait les petites filles de se laisser abuser par leur père. Ce tournant majeur eut un impact déterminant pour les années suivantes. Tu t'es presque rendue à la ligne d'arrivée de ce diplôme qui t'aurait permis d'exercer un métier et de rendre de précieux services à la société. Le hasard et une série d'événements malheureux ont voulu que tu rechutes, jusqu'à vouloir en finir avec la vie.

Alors que tu ne t'y attendais plus, c'est ta mère qui a repris contact avec toi. C'est avec elle que tu as entrepris cette longue remontée de l'abîme. Aujourd'hui, un autre défi t'attend: celui de donner un nouveau sens à ta vie malgré tout ce qui est arrivé. À la lecture de ce que tu m'as raconté, ton désir d'aider et de protéger les autres semble être demeuré intact. Peut-être est-ce là le sens de ta vie jusqu'ici? Peut-être est-ce là ton fil conducteur, le Fil d'Ariane de ta vie?

Si tu acceptes, nous allons t'aider par nos interventions à trouver une façon de concrétiser ce désir dans quelque chose de bien réel, un projet à ta portée, dans lequel tu pourras te réaliser et donner du sens à tes actions.

\section{VALIDATION DU FIL D'ARIANE}

On dirait que ce n'est pas de moi qu'il s'agit dans cette histoire. Pourtant, tous les faits sont exacts. Ça m'est bien arrivé, vous n'avez rien inventé, mais je n'arrive pas à comprendre que vous puissiez parler de moi en ces termes, alors que je me perçois tellement à l'opposé de ça!

On dirait que vous racontez mon histoire, mais avec des lunettes différentes de celles des autres. J'ai toujours cru que j'étais une ratée - mon père me l'a dit tellement souvent alors que vous me dites que je suis quelqu'un, je n'arrive pas à croire que vous pensez vraiment ça de moi... Mais ça me fait du bien de relire mon histoire, ça me donne même le goût de continuer. J'ai envie de la relire encore pour m'assurer que c'est bien de moi qu'il s'agit...

Dans sa dimension sens à la vie, la spiritualité constitue l'un des facteurs de résilience qui permettent à la personne en réadaptation de «rebondir et s'adapter en dépit de l'adversité» (Rutter, 1985). L'épisode de réadaptation peut devenir une expérience significative de croissance, si l'intervenant arrive à l'inscrire dans le projet de vie de l'usager et à lui donner du sens. Le développement du Fil d'Ariane par le Centre de réadaptation Estrie s'inscrit dans cette perspective positive.

\section{Bibliographie}

AMERICAN PSYCHOLOGICAL ASSOCIATION (2010). "Road to resiliency », en ligne, <http://www.apa.org/helpcenter/ road-resilience.aspx/>, consulté le 201003-11.

BLACK, K. et M. LOBO (2008). "A conceptual review of family resilience factors ", Journal of Family Nursing, vol. 14, $\mathrm{n}^{\circ} 1$, p. 33-55.

BONANNO, G.A. (2004). «Loss, trauma, and human resilience», American Psychologist, vol. 59, n 1 , p. 20-28.

CHOUINARD, J. et J. TARDIF (2005). «Le Fil d'Ariane», document inédit, Sherbrooke, Centre de réadaptation Estrie.

CYRULNIK, B. (2008). Conférence prononcée lors du colloque "Résilience: intervention en contexte de réadaptation adaptation », Montréal, Groupe de recherche GIRAFE.

CYRULNIK, B. (1999). Un merveilleux malheur, Paris, Odile Jacob.

CONNOR, K.M. et R.T. DAVIDSON (2003). "Development of a new resiliency scale: The Connor-Davidson Resiliency Scale», Depression and Anxiety, $\mathrm{n}^{\circ}$ 18, p. 76-82.

DUMONT, C., M. GERVAIS, P. FOUGEYROLLAS et R. BERTRAND (2004). "Toward an explanatory model of social participation for adults with traumatic brain injury ", Journal of Head Trauma Rehabilitation, vol. 19, $\mathrm{n}^{\circ} 6$, p. 431-444.

DUNN, D.S. et S.B. DOUGHERTY (2005). "Prospects for a positive psychology of rehabilitation ", Rehabilitation Psychology, vol. 50, no 3, p. 305-311.

DYER, J.G. et T.M. MCGUINNESS (1996). "Resilience: Analysis of the concept», Archives of Psychiatric Nursing, $\mathrm{n}^{\mathrm{o}} 10$, p. 276-282.

FOUGEYROLLAS, P. (1998). Le Processus de production du handicap, Québec, Réseau international sur le Processus de production du handicap (RIPPH).

FRANKL, V. (2006). Découvrir un sens à sa vie, Montréal, Éd. de l'Homme.

FRAMPTON, S.B. et P.A. CHARMEL (2009). Putting Patients First: Best Practices in Patient-Centered Care, $2^{\mathrm{e}}$ édition, San Francisco, Jossey-Bass.

JACELON, C.S. (1997). "The trait and process of resilience», Journal of Advanced Nursing, $\mathrm{n}^{\circ} 25$, p. 123-129.

KING, G., T. CATHERS, E. BROWN, J.A. SPECHT, C. WILLOUGHBY, J. MILLER POLGAR, E. MACKINNON, L.K. SMITH et L. HAVENS (2003). "Turning points and protective processes in the lives of people with chronic disabilities", Qualitative Health Research, vol. 13, no 2, p. 184-206.
LACHAPELLE, P.-P. (2009). "Le cadre conceptuel PAR: le processus d'adaptation et de réadaptation de la personne (PAR) : son application en interventions médicosociales et psychosociales », document inédit, Montréal.

LAW, M., H. POLATAJKO, S. BAPTISTE et E. TOWNSEND (2002). "Core concepts of occupational therapy», dans E. TOWNSEND (dir.), Enabling Occupations: An Occupational Therapy Perspective, Ottawa, Canadian Association of Occupational Therapists, p. 29-56.

MATTINGLY, C. (2007). Healing Dramas and Clinical Plots: The Narrative Structure of Experience, New York, Cambridge University Press.

MCMILLEN, J.C. et C. LOVELAND-COOK (2003). "The positive by-products of spinal cord injury and their correlates", Rehabilitation Psychology, vol. 48, $\mathrm{n}^{\circ}$ 2, p. 77-85.

PLEDGER, C. (2003). «Discourse on disability and rehabilitation issues ", American Psychologist, vol. 58, $\mathrm{n}^{\circ}$ 4, p. 279-284.

RICHARDSON, G.E. (2002). "The metatheory of resilience and resiliency ", Journal of Clinical Psychology, vol. 58, no 3, p. 307321.

RUTTER, M. (1985). «Resiliency in the face of adversity: Protective factors and resistance to psychiatric disorders", British Journal of Psychiatry, no 147, p. 598-611.

SELIGMAN, M.E.P. et M. CSIKSZENTMIHALYI (2000). "Positive psychology: An introduction ", American Psychologist, vol. 55, no 1 , p. 5-14.

THOMPSON, N.J., J. COKER, J.S. KRAUSE et E. HENRY (2003). "Purpose in life as a mediator of adjustment after spinal cord injury ", Rehabilitation Psychology, vol. 48, $\mathrm{n}^{\circ} 2$, p. $100-108$.

WAGNILD, G. et H.M. YOUNG (1993). «Development and psychometric evaluation of the Resilience Scale », Journal of Nursing Measurement, vol. 1, n 2 , p. 165-178.

WHITE, B., S. DRIVER et A.-M. WARREN (2008). "Considering resilience in the rehabilitation of people with traumatic disabilities», Rehabilitation Psychology, vol. 53, $\mathrm{n}^{\mathrm{o}}$ 1, p. 9-17.

YALOM, I. D. (2008). Thérapie existentielle, Paris, Galaade.

\section{Notes}

1. Perceived self-efficacy: concept émanant de la théorie sociale de Bandura, impliquant une confiance en ses propres forces et capacités et la croyance en la capacité de réaliser ses projets et de répondre aux demandes de son environnement (Dumont et al., 2004).

\section{Disabling factors vs enabling factors.}

3. Plusieurs détails de l'histoire de cette femme ont été modifiés afin de ne pas permettre son identification. 